\title{
Giant Pyogenic Granuloma in a Patient with Chronic Lymphocytic Leukemia
}

\author{
Molly Plovanich ${ }^{\mathrm{a}}$ Hillary C. Tsibris ${ }^{\mathrm{c}}$ Christine G. Lian ${ }^{\mathrm{b}}$ \\ Arash Mostaghimi ${ }^{\mathrm{a}}$ \\ Departments of ${ }^{a}$ Dermatology and ${ }^{b}$ Pathology, Brigham and Women's Hospital and \\ Harvard Medical School, and ${ }^{\mathrm{C}} \mathrm{H}$ arvard Combined Dermatology Residency Program, \\ Boston, Mass., USA
}

\section{Key Words}

Giant pyogenic granuloma $\cdot$ Chronic lymphocytic leukemia

\begin{abstract}
Pyogenic granuloma, also known as lobular capillary hemangioma, is a common benign vascular proliferative lesion that can present at any age on the skin or mucous membranes. Most lesions do not exceed $2 \mathrm{~cm}$, but there are a handful of giant cutaneous pyogenic granulomas that have been reported, often in individuals with underlying immune dysfunction. Here, we report the first giant pyogenic granuloma in a patient with a hematological malignancy, chronic lymphocytic leukemia.

(c) 2014 S. Karger AG, Basel
\end{abstract}

\section{Introduction}

Pyogenic granuloma is a common benign vascular proliferative lesion of the skin and mucosa. First described in 1897 by Poncet and Dor, it was thought to represent a reactive response to an unidentified bacterium given the florid granulation tissue and inflammation on histology. However, no causal bacterium was identified, and in 1980, it was proposed that pyogenic granuloma was instead a vascular proliferative lesion, which could be best visualized by histology of the lesion's base [1]. Hence, the alternative name for pyogenic granuloma, lobular capillary hemangioma, was introduced. Here, we present a case of a giant cutaneous pyogenic granuloma in a patient with chronic lymphocytic leukemia (CLL). 
Plovanich et al.: Giant Pyogenic Granuloma in a Patient with Chronic Lymphocytic Leukemia

\section{Case Report}

A 52-year-old woman with CLL presented with a 1-month history of a rapidly expanding mass on her forearm. She reported intermittent bleeding and crusting, but denied pain, pruritus or frank pus. A week prior to the development of the mass, she had begun chemotherapy for CLL with rituximab, bendamustine and lenalidomide requiring multiple blood draws from her arm. Her medical history was otherwise unremarkable. She had no recent travel, animal exposures or new medications aside from chemotherapy.

Physical examination revealed a $4.7 \times 3.7 \mathrm{~cm}$ pedunculated, firm mass with a fleshy, erythematous stalk and a peripheral border of hemorrhagic crust on the volar surface of her left forearm (fig. 1a). Laboratory results were significant for pancytopenia with a white blood cell count of 800 cells $/ \mu \mathrm{l}$ (16\% neutrophils). Punch biopsy of the stalk was performed, which showed a histological picture most suggestive of reactive changes to trauma (fig. 1b). However, given concern that the biopsy was not representative of the entire lesion, full excision was recommended. The excision specimen demonstrated a polypoid lesion with vaguely lobulated vascular proliferation (fig. 1c). High magnification showed a lobulated proliferation of capillary-sized vessels (fig. 1d).

Postoperatively, the patient experienced wound dehiscence in the setting of persistent neutropenia. She was treated with wet-to-dry dressings, and her chemotherapy was held. Subsequently, her white blood cell count normalized, and her wound healed without a revised closure or skin graft.

\section{Discussion}

Pyogenic granuloma often presents as a solitary, red-purple nodule that undergoes rapid growth over weeks to months. Mucosal lesions are more common in women (2.6:1), particularly during pregnancy, pointing to estrogen as an important driving factor in their pathogenesis [2]. In contrast, cutaneous lesions have a slight male predominance (1.2:1) and are commonly found on the trunk and upper extremities in adults [2]. This divergent epidemiology of mucosal and cutaneous pyogenic granulomas suggests that these lesions may have partially distinct routes of pathogenesis.

In our case, the patient's pyogenic granuloma was $4.7 \times 3.7 \mathrm{~cm}$, which far exceeds the previously published average size of pyogenic granulomas, which ranges from 0.65 to $1.1 \mathrm{~cm}$ $[3,4]$. There are a handful of giant cutaneous pyogenic granulomas that have been reported in the past, often in individuals with underlying immune dysfunction, but never in a patient with CLL $[5,6]$.

In making the diagnosis of pyogenic granuloma, it is important to exclude a more sinister diagnosis, such as amelanotic melanoma, metastatic carcinoma, leukemia cutis, squamous cell carcinoma, angiosarcoma, bacillary angiomatosis and fungal infection, which can be done using light microscopy. In our patient, given her history of progressive CLL, it was important to exclude leukemia cutis and squamous cell carcinoma, particularly given her eight-fold increased risk for developing a squamous cell carcinoma with a poor outcome $[7,8]$. Thus, heightened suspicion for these diagnoses contributed to the decision to pursue excisional biopsy despite her inconclusive punch biopsy to definitely exclude these diagnoses. The characteristic histopathological findings of pyogenic granulomas include a lobular arrangement of proliferating capillaries separated by myxoid or fibrous connective tissue septa. Often, this architecture is obscured by edema, hemorrhage, inflammation and ulceration, much like our patient's pathology showed. Immunohistochemistry is rarely 
Plovanich et al.: Giant Pyogenic Granuloma in a Patient with Chronic Lymphocytic Leukemia

performed, but would reveal positive staining for vascular markers CD31, CD34 and factor VIII. Glucose transporter 1 staining is negative, thereby distinguishing it from juvenile hemangioma.

There are numerous treatments available for pyogenic granulomas, including surgical and medical options [9]. Generally, the choice of treatment depends on the location, size and shape of the underlying lesion. Certain practices are associated with lower rates of recurrence, including surgical excision and topical imiquimod therapy, but there are very few randomized controlled trials directly comparing available therapies [9].

At present, the pathogenesis of pyogenic granuloma is unknown. Reported triggers include antecedent trauma, pregnancy and certain drugs, including acitretin, HIV protease inhibitors, EGFR inhibitors, docetaxel, capecitabine and rituximab [10, 11]. Interestingly, drug-associated pyogenic granulomas tend to have a periungual and subungual distribution [10]. Recent insights from gene expression profiling identify FLT4 (a specific VEGF receptor), the nitric oxide pathway and vascular injury and repair pathways as specifically upregulated in pyogenic granulomas compared to other vascular proliferative lesions [12]. How the molecular underpinnings connect to the diverse set of reported triggers remains unclear.

This case highlights an unusual presentation of a pyogenic granuloma given the shear size of the lesion. Contributing factors may include immune dysfunction due to CLL, persistent neutropenia and trauma sustained due to multiple blood draws to her left forearm. Although there is one reported case of multiple, small periungual and subungual pyogenic granulomas following rituximab exposure, the appearance and distribution of these lesions were unlike our patient's, making rituximab a less likely contributing factor [11]. However, given the small number of giant pyogenic granulomas reported to date, it is difficult to exclude any associations with medications. Interestingly, in the handful of giant cutaneous pyogenic granulomas reported, the majority of hosts had underlying immune dysfunction, which may be an important risk factor for the development of such large pyogenic granulomas.

\section{Disclosure Statement}

The authors report no conflicts of interest. No funding or support was received.

\section{References}

1 Mills SE, Cooper PH, Fechner RE: Lobular capillary hemangioma: the underlying lesion of pyogenic granuloma. A study of 73 cases from the oral and nasal mucous membranes. Am J Surg Pathol 1980;4:470479.

2 Harris MN, Desai R, Chuang TY, Hood AF, Mirowski GW: Lobular capillary hemangiomas: an epidemiologic report, with emphasis on cutaneous lesions. J Am Acad Dermatol 2000;42:1012-1016.

-3 Patrice SJ, Wiss K, Mulliken JB: Pyogenic granuloma (lobular capillary hemangioma): a clinicopathologic study of 178 cases. Pediatr Dermatol 1991;8:267-276.

4 Leyden JJ, Master GH: Oral cavity pyogenic granuloma. Arch Dermatol 1973;108:226-228.

5 Nthumba PM: Giant pyogenic granuloma of the thigh: a case report. J Med Case Rep 2008;2:95.

6 Dissemond J, Grabbe S: Giant pyogenic granuloma. CMAJ 2008;178:25-26.

7 Manusow D, Weinerman BH: Subsequent neoplasia in chronic lymphocytic leukemia. JAMA 1975;232:267269.

8 Velez NF, Karia PS, Vartanov AR, Davids MS, Brown JR, Schmults CD: Association of advanced leukemic stage and skin cancer tumor stage with poor skin cancer outcomes in patients with chronic lymphocytic leukemia. JAMA Dermatol 2014;150:280-287.

-9 Lee J, Sinno H, Tahiri Y, Gilardino MS: Treatment options for cutaneous pyogenic granulomas: a review. J Plast Reconstr Aesthet Surg 2011;64:1216-1220. 
Plovanich et al.: Giant Pyogenic Granuloma in a Patient with Chronic Lymphocytic Leukemia

10 Piraccini BM, Bellavista S, Misciali C, Tosti A, de Berker D, Richert B: Periungual and subungual pyogenic granuloma. Br J Dermatol 2010;163:941-953.

11 Wollina U: Multiple eruptive periungual pyogenic granulomas during anti-CD20 monoclonal antibody therapy for rheumatoid arthritis. J Dermatol Case Rep 2010;4:44-46.

$\checkmark 12$ Godfraind C, Calicchio ML, Kozakewich H: Pyogenic granuloma, an impaired wound healing process, linked to vascular growth driven by FLT4 and the nitric oxide pathway. Mod Pathol 2013;26:247-255. 


\section{Case Reports in Dermatology}

\begin{tabular}{l|l}
\hline Case Rep Dermatol 2014;6:227-231 \\
\hline DOI: $10.1159 / 000367935$ & $\begin{array}{l}\text { (c) 2014 S. Karger AG, Basel } \\
\text { www.karger.com/cde }\end{array}$
\end{tabular}

Plovanich et al.: Giant Pyogenic Granuloma in a Patient with Chronic Lymphocytic Leukemia
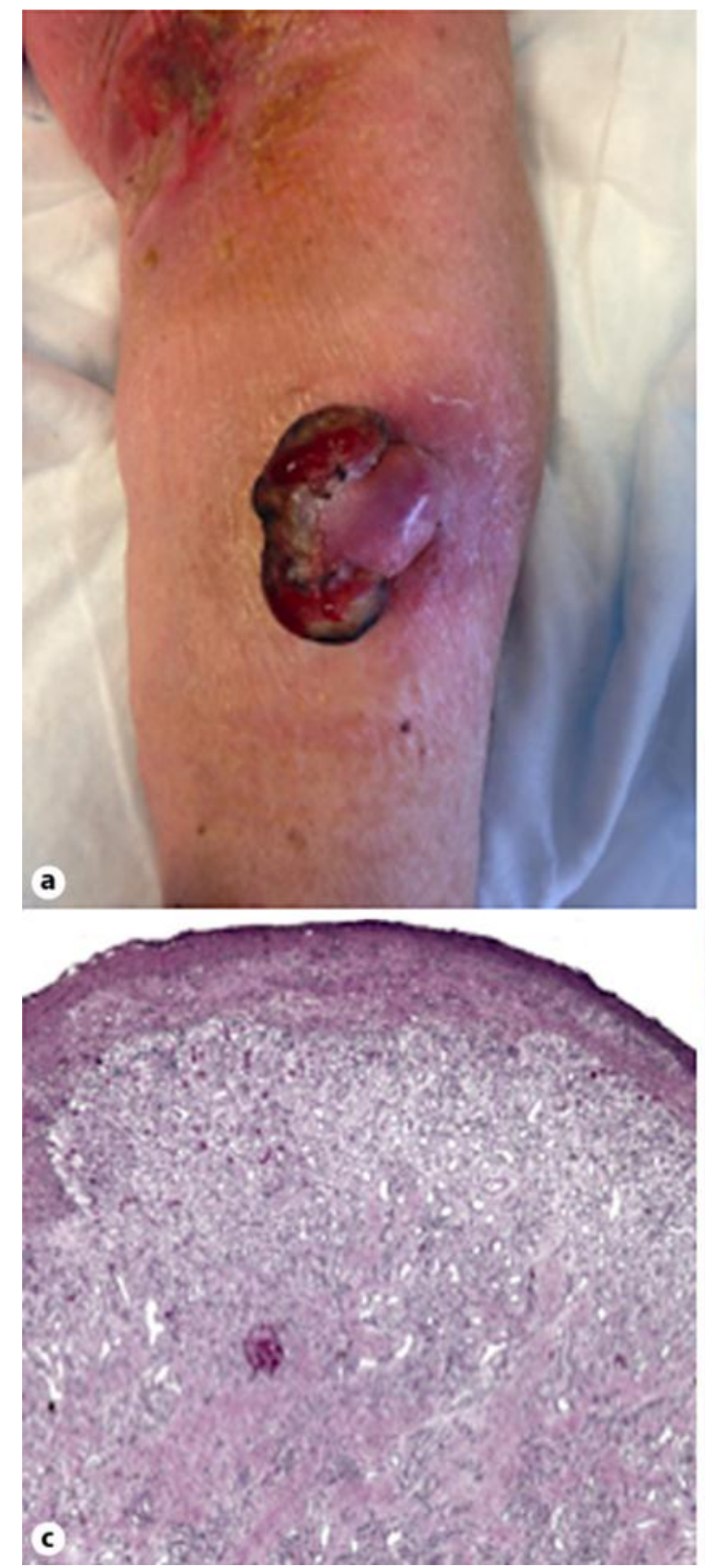
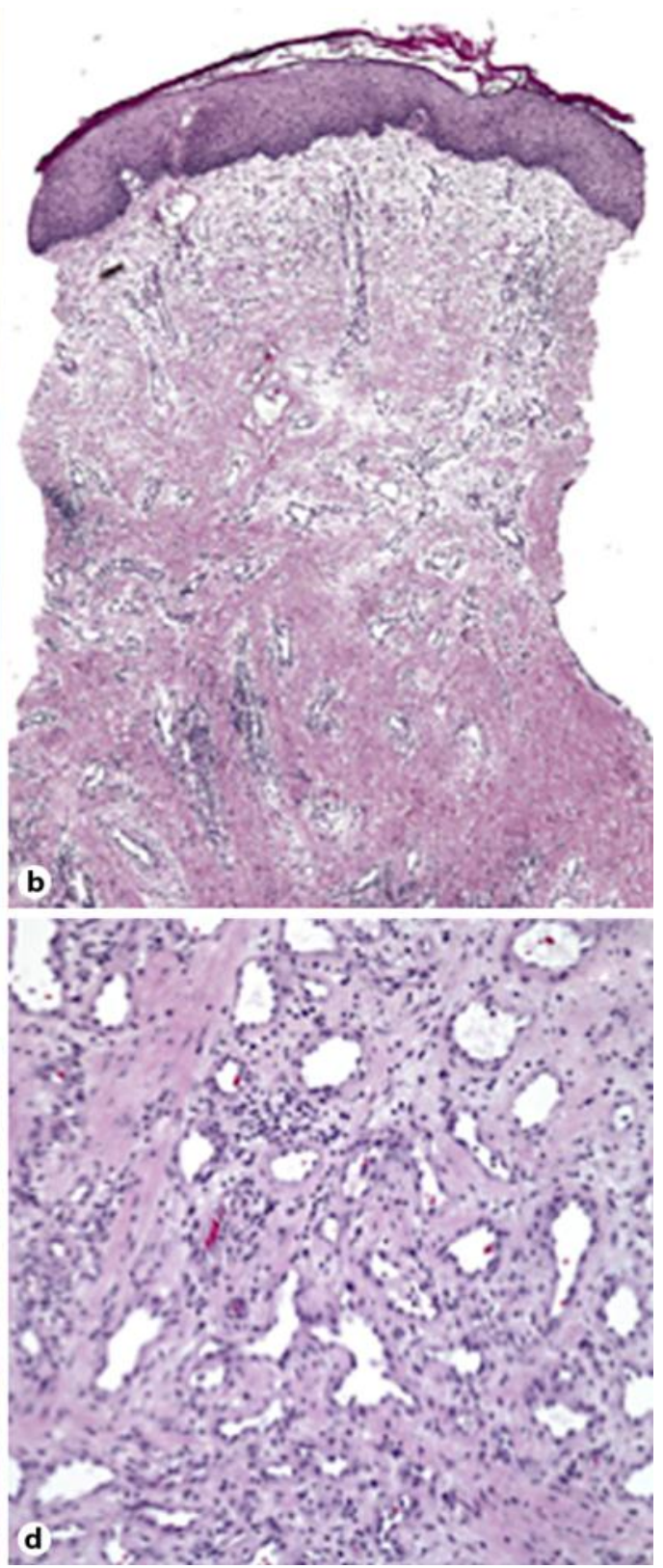

Fig. 1. a The pedunculated mass on the left volar forearm. b Histopathology of the punch biopsy showed slightly acanthotic epidermis with dermal fibrosis and vascular proliferation with mixed inflammatory infiltrate, most consistent with reactive changes to trauma (hematoxylin-eosin, original magnification $\times 4$ ). c Although no evidence of malignancy was identified in the biopsy specimen, excision was recommended given the large size of the lesion and clinical suspicion for malignancy that was not visualized on the biopsy. The excision specimen demonstrated a large ulcerated, polypoid lesion with dermal edema and hemorrhage and vaguely lobulated vascular proliferation (hematoxylin-eosin, original magnification $\times 10$ ). d High magnification of the excisional specimen demonstrated lobulated proliferation of capillary-sized vessels with small indistinct lumina. No cytological atypia was present (hematoxylin-eosin, original magnification $\times 40$ ). 